\title{
COMPOSITE FERMIONS AND THE FRACTIONAL QUANTUM HALL EFFECT
}

\author{
A. WóJs ${ }^{a b}$ AND J.J. QUINN ${ }^{a}$ \\ ${ }^{a}$ Department of Physics, University of Tennessee, Knoxville, Tennessee 37996, USA \\ ${ }^{b}$ Institute of Physics, Wrocław University of Technology \\ Wybrzeże Wyspiańskiego 27, 50-370 Wrocław, Poland
}

The mean field composite fermion picture successfully predicts low lying states of fractional quantum Hall systems. This success cannot be attributed to a cancellation between the Coulomb and Chern-Simons interactions beyond the mean field and solely depends on the short-range of the Coulomb pseudopotential in the lowest Landau level. The class of pseudopotentials for which the mean field composite fermion picture can be applied is defined. The success or failure of the mean field composite fermion picture in various systems (electrons in excited Landau levels, Laughlin quasiparticles, charged magnetoexcitons) is explained.

PACS numbers: 71.10.Pm, 73.20.Dx, 73.40.Hm

\section{Introduction}

The quantum Hall effect (QHE) [1, 2], i.e. the quantization of Hall conductance of a two-dimensional electron gas (2DEG) in high magnetic fields at certain filling factors $\nu\left(\nu^{-1}\right.$ is the number of single particle states in a Landau level (LL) per electron), signals the appearance of (incompressible) non-degenerate ground states (GS's) separated from the continuum of excited states by a finite gap. At integer $\nu=1,2 \ldots$ (IQHE), the excitation gap is the single particle cyclotron gap, while at fractional $\nu=1 / 3,1 / 5,2 / 5 \ldots$ (FQHE) electrons partially fill a degenerate (lowest) LL and the formation of incompressible GS's is a many-body phenomenon revealing the unique properties of the Coulomb interaction of electrons in the lowest LL $[3,4]$.

In the mean field (MF) composite fermion (CF) picture $[5,6]$, in a 2DEG of density $n$ at a strong magnetic field $B$, each electron binds an even number $2 p$ of magnetic flux quanta $\phi_{0}=h c / e$ (in form of an infinitely thin flux tube) forming a CF. Because of the Pauli exclusion principle, the magnetic field confined into a flux tube within one CF has no effect on the motion of other CF's, and the average effective magnetic field $B^{*}$ seen by CF's is reduced, $B^{*}=B-2 p \phi_{0} n$. 
Because $B^{*} \nu^{*}=B \nu=n \phi_{0}$, the relation between the electron and CF filling factors is

$$
\left(\nu^{*}\right)^{-1}=\nu^{-1}-2 p
$$

Since the low band of energy levels of the original (interacting) 2DEG has similar structure to that of the noninteracting CF's in a uniform effective field $B^{*}$, it was proposed [5] that the Coulomb charge-charge and Chern-Simons (CS) charge-flux interactions beyond the MF largely cancel one another, and the original strongly interacting system of electrons is converted into one of weakly interacting CF's. Consequently, the FQHE of electrons was interpreted as the IQHE of CF's.

Although the MFCF picture correctly predicts the structure of low energy spectra of FQH systems, the energy scale it uses (the CF cyclotron energy $\hbar \omega_{c}^{*}$ ) is totally irrelevant. Moreover, since the characteristic energies of the CS $\left(\hbar \omega_{\mathrm{c}}^{*} \propto B\right)$ and Coulomb $\left(e^{2} / \lambda \propto \sqrt{B}\right.$, where $\lambda$ is the magnetic length) interactions between fluctuations beyond MF scale differently with the magnetic field, the reason for its success cannot be found in originally suggested cancellation between those interactions. Since the MFCF picture is commonly used to interpret various numerical and experimental results, it is very important to understand why and under what conditions it is correct.

In this paper, we use the pseudopotential formalism $[7,8]$ to study the $\mathrm{FQH}$ systems. It is shown that the form of the pseudopotential $V\left(L^{\prime}\right)$ [pair energy vs. pair angular momentum] rather than of the interaction potential $V(r)$, is responsible for the incompressibility of FQH states. The idea of fractional parentage [9] is used to characterize many-body states by the ability of electrons to avoid pair states with largest repulsion. The condition on the form of $V\left(L^{\prime}\right)$ necessary for the occurrence of FQH states is given, which defines the class of short-range (SR) pseudopotentials to which MFCF picture can be applied. As an example, we explain the success or failure of MFCF predictions for the systems of electrons in the lowest and excited LL's, Laughlin quasiparticles (QP's) in the hierarchy picture of FQH states [10,11], and charged excitons in a 2D electron-hole plasma [12].

\section{Numerical studies on the Haldane sphere}

Because of the LL degeneracy, the electron-electron interaction in the FQH states cannot be treated perturbatively, and the exact (numerical) diagonalization techniques have been commonly used. In order to model an infinite 2DEG by a finite (small) system that can be handled numerically, it is very convenient to confine $N$ electrons to a surface of a (Haldane) sphere of radius $R$, with the normal magnetic field $B$ produced by a magnetic monopole of integer strength $2 S$ (total flux of $4 \pi B R^{2}=2 S \phi_{0}$ ) in the center [10]. The obvious advantages of such geometry is the absence of an edge and preserving full 2D symmetry of a 2DEG (good quantum numbers are the total angular momentum $L$ and its projection $M$ ). The numerical experiments in this geometry have shown that even relatively small systems that can be solved exactly on a small computer behave in many ways like an infinite $2 \mathrm{DEG}$, and a number of parameters of a 2DEG (e.g. characteristic excitation energies) can be obtained from such small scale calculations. 
The single particle states on a Haldane sphere (monopole harmonics) are labeled by angular momentum $l$ and its projection $m$ [13]. The energies, $\varepsilon_{l}=$ $\hbar \omega_{c}\left[l(l+1)-S^{2}\right] / 2 S$, fall into degenerate shells and the $n$-th shell $(n=l-|S|=$ $0,1, \ldots)$ corresponds to the $n$-th LL. For the FQH states at filling factor $\nu<1$, only the lowest, spin polarized LL needs to be considered.

The object of numerical studies is to diagonalize the electron-electron interaction Hamiltonian $H$ in the space of degenerate antisymmetric $N$ electron states of a given (lowest) LL. Although matrix $H$ is easily block diagonalized into blocks with specified $M$, the exact diagonalization becomes difficult (matrix dimension over $\left.10^{6}\right)$ for $N>10$ and $2 S>27(\nu=1 / 3)$ [8]. Typical results for ten electrons at filling factors near $\nu=1 / 3$ are presented in Fig. 1. Energy $E$, plotted as a function of $L$ in the magnetic units, includes shift $-(N e)^{2} / 2 R$ due to charge compensating background. There is always one or more $L$ multiplets (marked with open circles) forming a low energy band separated from the continuum by a gap. If the lowest band consists of a single $L=0$ GS (Fig. 1d), it is expected to be incompressible in the thermodynamic limit (for $N \rightarrow \infty$ at the same $\nu$ ) and an infinite 2DEG at this filling factor is expected to exhibit the FQHE.

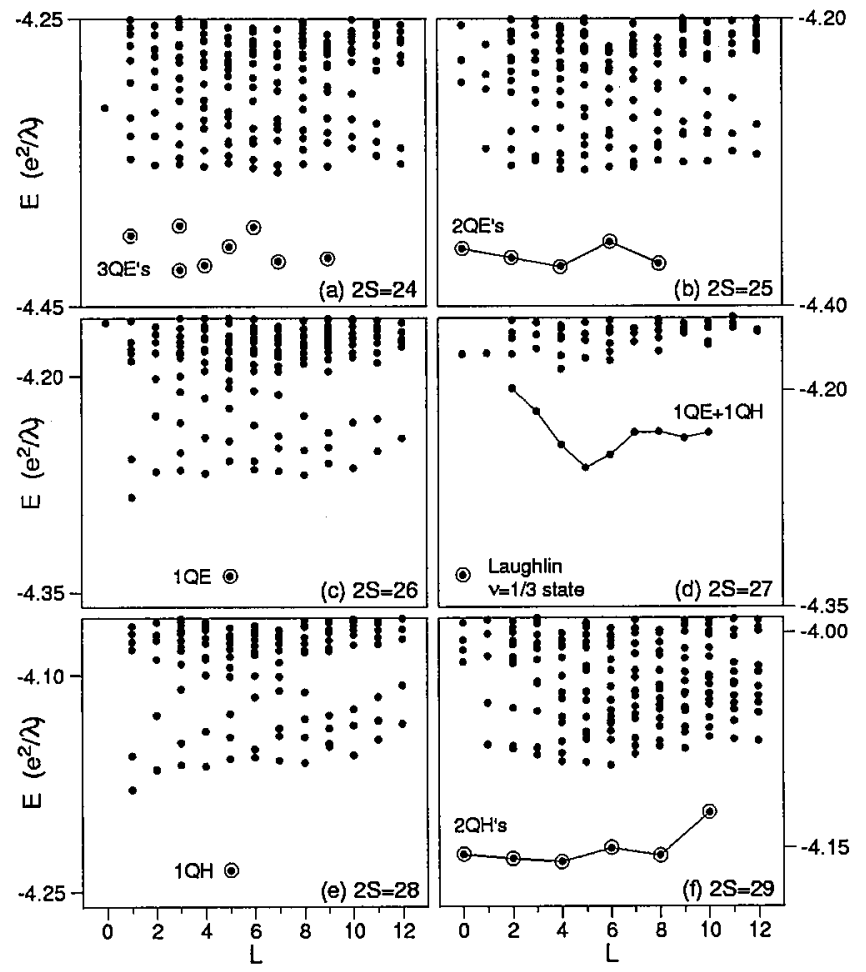

Fig. 1. Energy spectra of ten electrons in the lowest LL at the monopole strength $2 S$ between 24 and 29. Open circles mark lowest energy bands with fewest CF QP's. 
The MFCF interpretation of the spectra in Fig. 1 is the following. The effective magnetic monopole strength seen by CF's is $[5,8]$

$$
2 S^{*}=2 S-2 p(N-1),
$$

and the angular momenta of lowest CF shells (CF LL's) are $l_{n}^{*}=\left|S^{*}\right|+n$ [14]. At $2 S=27, l_{0}^{*}=9 / 2$ and ten CF's fill completely the lowest CF shell $(L=0$ and $\nu^{*}=1$ ). The excitations of the $\nu^{*}=1$ CF GS involve an excitation of at least one $\mathrm{CF}$ to a higher CF LL, and thus (if the CF-CF interaction is weak on the scale of $\hbar \omega_{\mathrm{c}}^{*}$ ) the $\nu^{*}=1$ GS is incompressible and so is the Laughlin [3] $\nu=1 / 3$ GS of underlying electrons. The lowest lying excited states contain a pair of QP's: a quasihole $(\mathrm{QH})$ with $l_{\mathrm{QH}}=l_{0}^{*}=9 / 2$ in the lowest CF LL and a quasielectron (QE) with $l_{\mathrm{QE}}=\mathrm{l}_{1}^{*}=11 / 2$ in the first excited one. The allowed angular momenta of such pair are $L=1,2, \ldots, 10$. The $L=1$ state usually has high energy and the states with $L \geq 2$ form a well defined band with a magnetoroton minimum at a finite value of $L$. The lowest $\mathrm{CF}$ states at $2 S=26$ and 28 contain a single $\mathrm{QE}$ and a single $\mathrm{QH}$, respectively (in the $\nu^{*}=1 \mathrm{CF}$ state, i.e. the $\nu=1 / 3$ electron state), both with $l_{\mathrm{QP}}=5$, and the excited states will contain additional QE-QH pairs. At $2 S=25$ and 29 the lowest bands correspond to a pair of QP's, and the values of energy within those bands define the QP-QP interaction pseudopotential $V_{\mathrm{QP}}$. At $2 S=25$ there are two QE's each with $l_{\mathrm{QE}}=9 / 2$ and the allowed angular momenta (of two identical fermions) are $L=0,2,4,6$, and 8 , while at $2 S=29$ there are two QH's each with $l_{\mathrm{QH}}=11 / 2$ and $L=0,2,4,6,8$, and 10. Finally, at $2 S=24$, the lowest band contains three QE's each with $l_{\mathrm{QE}}=4$ in the Laughlin $\nu=1 / 3$ state (in the Fermi liquid picture, interacting with one another through $\left.V_{\mathrm{QE}}[15]\right)$ and $L=1,3^{2}, 4,5,6,7$, and 9 .

\section{Pseudopotential approach}

The two-body interaction Hamiltonian $H$ can be expressed as

$$
\widehat{H}=\sum_{i<j} \sum_{L^{\prime}} V\left(L^{\prime}\right) \widehat{\mathcal{P}}_{i j}\left(L^{\prime}\right),
$$

where $V\left(L^{\prime}\right)$ is the interaction pseudopotential [7] and $\widehat{\mathcal{P}}_{i j}\left(L^{\prime}\right)$ projects onto the subspace with angular momentum of pair $i j$ equal to $L^{\prime}$. For electrons confined to a LL, $L^{\prime}$ measures the average squared distance $d^{2}$ [8],

$$
\frac{\widehat{d}^{2}}{R^{2}}=2+\frac{S^{2}}{l(l+1)}\left[2-\frac{\widehat{L}^{\prime 2}}{l(l+1)}\right],
$$

and larger $L^{\prime}$ corresponds to smaller separation. Due to the confinement of electrons to one (lowest) LL, interaction potential $V(r)$ enters Hamiltonian $H$ only through a small number of pseudopotential parameters $V(2 l-\mathcal{R})$, where $\mathcal{R}$, relative pair angular momentum, is an odd integer.

In Fig. 2 we compare the Coulomb pseudopotentials $V\left(L^{\prime}\right)$ calculated for a pair of electrons on the Haldane sphere each with $l=5,15 / 2,10$, and $25 / 2$, in the lowest and first excited LL. For the reason that will become clear later, $V\left(L^{\prime}\right)$ is plotted as a function of $L^{\prime}\left(L^{\prime}+1\right)$. All pseudopotentials in Fig. 2 increase with increasing $L^{\prime}$. If $V\left(L^{\prime}\right)$ increased very quickly with increasing $L^{\prime}$ (we define ideal 
SR repulsion as: $\mathrm{d} V_{\mathrm{SR}} / \mathrm{d} L^{\prime} \gg 0$ and $\mathrm{d}^{2} V_{\mathrm{SR}} / \mathrm{d} L^{2} \gg 0$ ), the low lying many-body states would be the ones maximally avoiding pair states with largest $L^{\prime}[7,8]$. At filling factor $\nu=1 / m$ ( $m$ is odd) the many-body Hilbert space contains exactly one multiplet in which all pairs completely avoid states with $L^{\prime}>2 l-m$. This multiplet is the $L=0$ incompressible Laughlin state [3] and it is an exact GS of $V_{\mathrm{SR}}$.

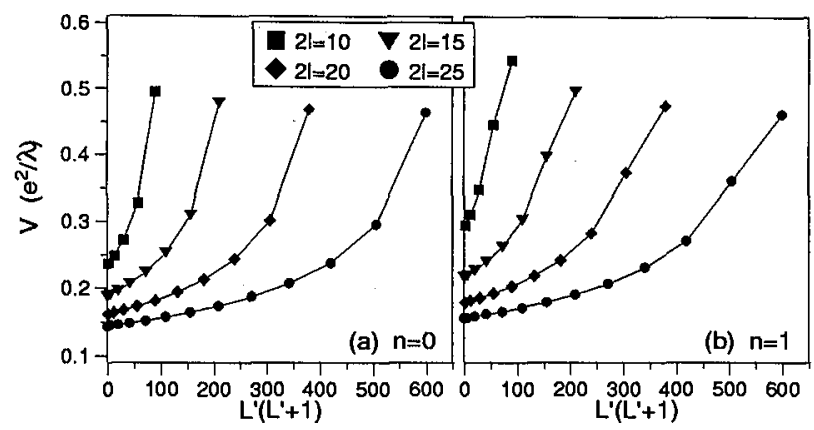

Fig. 2. Pseudopotentials $V$ of the Coulomb interaction in the lowest (a), and first excited LL (b) as a function of squared pair angular momentum $L^{\prime}\left(L^{\prime}+1\right)$. Squares $(l=5)$, triangles $(l=15 / 2)$, diamonds $(l=10)$, and circles $(l=25 / 2)$ mark data for different $S=l+n$.

The ability of electrons in a given many-body state to avoid strongly repulsive pair states can be conveniently described using the idea of fractional parentage $[8,9]$. An antisymmetric state $\left|l^{N}, L \alpha\right\rangle$ of $N$ electrons each with angular momentum $l$ that are combined to give total angular momentum $L$ can be written as

$$
\left|l^{N}, L \alpha\right\rangle=\sum_{L^{\prime}} \sum_{L^{\prime \prime} \alpha^{\prime \prime}} G_{L \alpha, L^{\prime \prime} \alpha^{\prime \prime}}\left(L^{\prime}\right)\left|l^{2}, L^{\prime} ; l^{N-2}, L^{\prime \prime} \alpha^{\prime \prime} ; L\right\rangle .
$$

Here $\left|l^{2}, L^{\prime} ; l^{N-2}, L^{\prime \prime} \alpha^{\prime \prime} ; L\right\rangle$ denote product states in which $l_{1}=l_{2}=l$ are added to obtain $L^{\prime}, l_{3}=l_{4}=\ldots=l_{N}=l$ are added to obtain $L^{\prime \prime}$ (different $L^{\prime \prime}$ multiplets are distinguished by a label $\alpha^{\prime \prime}$ ), and finally $L^{\prime}$ is added to $L^{\prime \prime}$ to obtain $L$. The state $\left|l^{N}, L \alpha\right\rangle$ is totally antisymmetric, and states $\left|l^{2}, L^{\prime} ; l^{N-2}, L^{\prime \prime} \alpha^{\prime \prime} ; L\right\rangle$ are antisymmetric under interchange of particles 1 and 2, and under interchange of any pair of particles $3,4, \ldots N$. The factor $G_{L \alpha, L^{\prime \prime} \alpha^{\prime \prime}}\left(L^{\prime}\right)$ is called the coefficient of fractional grand parentage (CFGP). The two-particle interaction matrix element expressed through CFGP's is

$$
\begin{aligned}
& \left\langle l^{N}, L \alpha|V| l^{N}, L \beta\right\rangle= \\
& \frac{N(N-1)}{2} \sum_{L^{\prime}} \sum_{L^{\prime \prime} \alpha^{\prime \prime}} G_{L \alpha, L^{\prime \prime} \alpha^{\prime \prime}}\left(L^{\prime}\right) G_{L \beta, L^{\prime \prime} \alpha^{\prime \prime}}\left(L^{\prime}\right) V\left(L^{\prime}\right),
\end{aligned}
$$

and expectation value of energy is

$$
E_{\alpha}(L)=\frac{N(N-1)}{2} \sum_{L^{\prime}} \mathcal{G}_{L \alpha}\left(L^{\prime}\right) V\left(L^{\prime}\right)
$$


where the coefficient

$$
\mathcal{G}_{L \alpha}\left(L^{\prime}\right)=\sum_{L^{\prime \prime} \alpha^{\prime \prime}}\left|G_{L \alpha, L^{\prime \prime} \alpha^{\prime \prime}}\left(L^{\prime}\right)\right|^{2}
$$

gives the probability that pair $i j$ is in the state with $L^{\prime}$.

\section{Energy spectra of short-range pseudopotentials}

The very good description of actual GS's of a 2DEG at fillings $\nu=1 / m$ by the Laughlin wave function (overlaps typically larger that 0.99 ) and the success of the MFCF picture at $\nu<1$ both rely on the fact that pseudopotential of the Coulomb repulsion in the lowest $L L$ falls into the same class of SR pseudopotentials as $V_{\mathrm{SR}}$. Due to a huge difference between all parameters $V_{\mathrm{SR}}\left(\mathrm{L}^{\prime}\right)$, the corresponding many-body Hamiltonian has the following hidden symmetry: the Hilbert space $\mathcal{H}$ contains eigensubspaces $\mathcal{H}_{p}$ of states with $\mathcal{G}\left(L^{\prime}\right)=0$ for $L^{\prime}>2(l-p)$, i.e. with $L^{\prime}<2(l-p)$. Hence, $\mathcal{H}$ splits into subspaces $\widetilde{\mathcal{H}}_{p}=\mathcal{H}_{p} \backslash \mathcal{H}_{p+1}$, containing states that do not have grand parentage from $L^{\prime}>2(l-p)$, but have some grand parentage from $L^{\prime}=2(l-p)-1$,

$$
\mathcal{H}=\tilde{\mathcal{H}}_{0} \oplus \tilde{\mathcal{H}}_{1} \oplus \tilde{\mathcal{H}}_{2} \oplus \ldots
$$

The subspace $\tilde{\mathcal{H}}_{p}$ is not empty (some states with $L^{\prime}<2(l-p)$ can be constructed) at filling factors $\nu \leq(2 p+1)^{-1}$. Since the energy of states from each subspace $\tilde{\mathcal{H}}_{p}$ is measured on a different scale of $V(2(l-p)-1)$, the energy spectrum splits into bands corresponding to those subspaces. The energy gap between the $p$-th and $(p+1)$-st bands is of the order of $V(2(l-p)-1)-V(2(l-p-1)-1)$, and hence the largest gap is that between the 0th band and the 1st band, the next largest is that between the 1st band and 2nd band, etc.

Figure 3 demonstrates on the example of four electrons to what extent this hidden symmetry holds for the Coulomb pseudopotential in the lowest LL. The subspaces $\mathcal{H}_{p}$ are identified by calculating CFGP's of all states. They are not exact eigenspaces of the Coulomb interaction, but the mixing between different $\mathcal{H}_{p}$ is weak and the coefficients $\mathcal{G}\left(L^{\prime}\right)$ for $L^{\prime}>2(l-p)$ (which vanish exactly in exact subspaces $\mathcal{H}_{p}$ ) are indeed much smaller in states marked with a given $p$ than in all other states. For example, for $2 l=11, \mathcal{G}(10)<0.003$ for states marked with full circled, and $\mathcal{G}(10)>0.1$ for all other states (squares).

Note that the set of angular momentum multiplets which form subspace $\tilde{\mathcal{H}}_{p}$ of $N$ electrons each with angular momentum $l$ is always the same as the set of multiplets in subspace $\tilde{\mathcal{H}}_{p+1}$ of $N$ electrons each with angular momentum $l+(N-1)$. When $l$ is increased by $N-1$, an additional band appears at high energy, but the structure of the low energy part of the spectrum is completely unchanged. For example, all three allowed multiplets for $l=5 / 2(L=0,2$, and 4$)$ form the lowest energy band for $l=11 / 2,17 / 2$, and $23 / 2$, where they span the $\widetilde{\mathcal{H}}_{1}$, $\widetilde{\mathcal{H}}_{2}$, and $\widetilde{\mathcal{H}}_{3}$ subspace, respectively. Similarly, the first excited band for $l=11 / 2$ is repeated for $l=17 / 2$ and $23 / 2$, where it corresponds to $\widetilde{\mathcal{H}}_{1}$ and $\widetilde{\mathcal{H}}_{2}$ subspace, respectively.

Let us stress that the fact that identical sets of multiplets occur in subspace $\widetilde{\mathcal{H}}_{p}$ for a given $l$ and in subspace $\widetilde{\mathcal{H}}_{q+1}$ for $l$ replaced by $l+(N-1)$, does not depend 


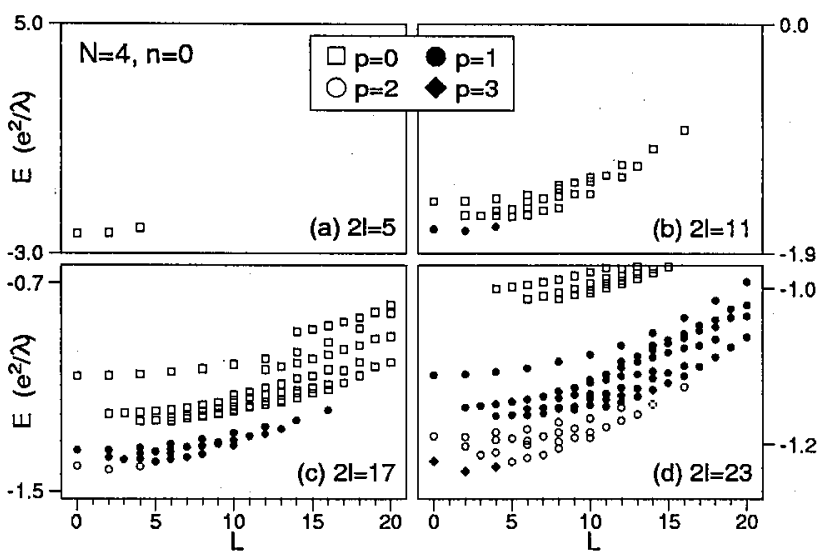

Fig. 3. Energy spectra of four electrons in the lowest LL each with angular momentum $l=5 / 2(\mathrm{a}), l=11 / 2(\mathrm{~b}), l=17 / 2(\mathrm{c})$, and $l=23 / 2(\mathrm{~d})$. Different subspaces $\mathcal{H}_{p}$ are marked with squares $(p=0)$, full circles $(p=1)$, open circles $(p=2)$, and diamonds $(p=3)$.

on the form of interaction, and follows solely from the rules of addition of angular momenta of identical fermions. However, if the interaction pseudopotential has SR, then: (i) $\widetilde{\mathcal{H}}_{p}$ are interaction eigensubspaces; (ii) energy bands corresponding to $\widetilde{\mathcal{H}}_{p}$ with higher $p$ lie below those of lower $p$; (iii) spacing between neighboring bands is governed by a difference between appropriate pseudopotential coefficients; and (iv) wave functions and structure of energy levels within each band are insensitive to the details of interaction. Replacing $V_{\mathrm{SR}}$ by a pseudopotential that increases more slowly with increasing $L^{\prime}$ leads to: (v) coupling between subspaces $\widetilde{\mathcal{H}}_{p} ;$ (vi) mixing, overlap, or even order reversal of bands; (vii) deviation of wave functions and the structure of energy levels within bands from those of the hard core repulsion (and thus their dependence on details of the interaction pseudopotential). The numerical calculations for the Coulomb pseudopotential in the lowest LL show (to a large extent) all SR properties (i)-(iv), and virtually no effects (v)-(vii), characteristic of "non SR" pseudopotentials.

The reoccurrence of $L$ multiplets forming the low energy band when $l$ is replaced by $l \pm(N-1)$ has the following crucial implication. In the lowest LL, the lowest energy ( $p$-th) band of the $N$ electron spectrum at the monopole strength $2 S$ contains $L$ multiplets which are all the allowed $N$ electron multiplets at $2 S-$ $2 p(N-1)$. But $2 S-2 p(N-1)$ is just $2 S^{*}$, the effective monopole strength of CF's! The MFCS transformation which binds $2 p$ fluxes (vortices) to each electron selects the same $L$ multiplets from the entire spectrum as does the introduction of a hard core, which forbids a pair of electrons to be in a state with $L^{\prime}>2(l-p)$.

\section{Definition of short-range pseudopotential}

A useful operator identity relates total $(L)$ and pair $\left(\widehat{L}_{i j}\right)$ angular momenta [8]

$$
\sum_{i<j} \widehat{L}_{i j}^{2}=\widehat{L}^{2}+N(N-2) \widehat{l}^{2} .
$$


It implies that interaction given by a pseudopotential $V\left(L^{\prime}\right)$ that is linear in $\widehat{L}^{\prime 2}$ (e.g. the harmonic repulsion within each LL; see Eq. (4)) is degenerate within each $L$ subspace and its energy is a linear function of $L(L+1)$. The many body GS has the lowest available $L$ and is usually degenerate, while the state with maximum $L$ has the largest energy. Note that this result is opposite to the Hund rule valid for spherical harmonics, due to the opposite behavior of $V\left(L^{\prime}\right)$ for the FQH $(n=0$ and $l=S)$ and atomic $(S=0$ and $l=n)$ systems.

Deviations of $V\left(L^{\prime}\right)$ from a linear function of $L^{\prime}\left(L^{\prime}+1\right)$ lead to the level repulsion within each $L$ subspace, and the GS is no longer necessarily the state with minimum $L$. Rather, it is the state at a low $L$ whose multiplicity $N_{L}$ (number of different $L$ multiplets) is large. It is interesting to observe that the $L$ subspaces with relatively high $N_{L}$ coincide with the MFCF prediction. In particular, for a given $N$, they reoccur at the same $L$ 's when $l$ is replaced by $l \pm(N-1)$, and the set of allowed $L$ 's at a given $l$ is always a subset of the set at $l+(N-1)$.

As we said earlier, if $V\left(L^{\prime}\right)$ has SR, the lowest energy states within each $L$ subspace are those maximally avoiding large $L^{\prime}$, and the lowest band (separated from higher states by a gap) contains states in which a number of largest values of $L^{\prime}$ is avoided altogether. This property is valid for all pseudopotentials which increase more quickly than linearly as a function of $L^{\prime}\left(L^{\prime}+1\right)$. For $V_{\beta}\left(L^{\prime}\right)=$ $\left[L^{\prime}\left(L^{\prime}+1\right)\right]^{\beta}$, exponent $\beta>1$ defines the class of SR pseudopotentials, to which the MFCF picture can be applied. Within this class, the structure of low lying energy spectrum and the corresponding wave functions very weakly depend on $\beta$ and converge to those of $V_{\mathrm{SR}}$ for $\beta \rightarrow \infty$.

The extension of the SR definition to $V\left(L^{\prime}\right)$ that are not strictly in the form of $V_{\beta}\left(L^{\prime}\right)$ is straightforward. If $V\left(L^{\prime}\right)>V(2 l-m)$ for $L^{\prime}>2 l-m$ and $V\left(L^{\prime}\right)<V(2 l-m)$ for $L^{\prime}<2 l-m$ and $V\left(L^{\prime}\right)$ increases more quickly than linearly as a function of $L^{\prime}\left(L^{\prime}+1\right)$ in the vicinity of $L^{\prime}=2 l-m$, then pseudopotential $V\left(L^{\prime}\right)$ behaves like a SR one at filling factors near $\nu=1 / m$.

\section{Application to various pseudopotentials}

It follows from Fig. 2a that the Coulomb pseudopotential in the lowest LL satisfies the $\mathrm{SR}$ condition in the entire range of $L^{\prime}$; this is what validates the MFCF picture for filling factors $\nu \leq 1$. It also explains the formation of incompressible states of charged magnetoexcitons $\left(\mathrm{X}^{-}\right)$formed in the electron-hole plasma [12]. However, in a higher, $n$-th LL this is only true for $L^{\prime}<2(l-n)-1$ (see Fig. 2b for $n=1$ ) and the MFCF picture is valid only for $\nu_{n}$ (filling factor in the $n$-th LL) around and below $(2 n+3)^{-1}$. Indeed, the MFCF features in the ten electron energy spectra around $\nu=1 / 3$ (in Fig. 1) are absent for the same fillings of the $n=1 \mathrm{LL}[8]$.

One consequence of this is that the MFCF picture or the Laughlin-like wave function cannot be used to describe the reported [16] incompressible state at $\nu=$ $2+1 / 3=7 / 3\left(\nu_{1}=1 / 3\right)$. The correlations in the $\nu=7 / 3$ GS are different than at $\nu=1 / 3$; the origin of (apparent) incompressibility cannot be attributed to the formation of a Laughlin-like $\nu_{1}=1 / 3$ state (in which pair states with smallest average separation $d^{2}$ are avoided) on top of the $\nu=2$ state and connection 
between the excitation gap and the pseudopotential parameters is different. This is clearly visible in the dependence of the excitation gap $\Delta$ on the electron number $N$, plotted in Fig. 4 for $\nu=1 / 3$ and $1 / 5$ fillings of the lowest and first excited LL. The gaps for $\nu=1 / 5$ behave very similarly as a function of $N$ in both LL's, while it is not even possible to make a conclusive statement about degeneracy or incompressibility of the $\nu=7 / 3$ state based on our data for up to eleven electrons.

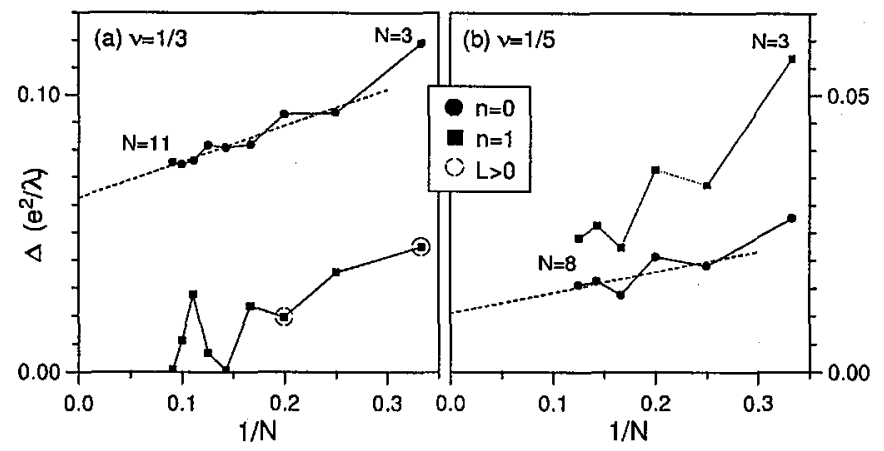

Fig. 4. Excitation gap $\Delta$ as a function of inverse electron number $1 / N$ for filling factors $\nu=1 / 3(\mathrm{a})$ and $1 / 5$ (b) in the $n=0$ (dots) and $n=1$ (squares) LL's. Open circles mark degenerate ground states $(L>0)$.

The SR criterion can be applied to the QP pseudopotentials to understand why QP's do not form incompressible states at all Laughlin filling factors $\nu_{\mathrm{QP}}=1 / \mathrm{m}$ in the hierarchy picture $[10,11]$ of $\mathrm{FQH}$ states. Lines in Fig. $1 \mathrm{~b}$ and f mark $V_{\mathrm{QE}}$ and $V_{\mathrm{QH}}$ for the Laughlin $\nu=1 / 3$ state of ten electrons. Clearly, the incompressible states with a large gap will be formed by QH's at $\nu_{\mathrm{QH}}=1 / 3$ and by QE's at $\nu_{\mathrm{QE}}=1$, explaining strong FQHE of the underlying electron system at the Jain $\nu=2 / 7$ and $2 / 5$ fractions, respectively. On the other hand, there is no FQHE at $\nu_{\mathrm{QH}}=1 / 5(\nu=4 / 13)$ or $\nu_{\mathrm{QE}}=1 / 3(\nu=4 / 11)$, and the gap above possibly incompressible $\nu_{\mathrm{QH}}=1 / 7(\nu=6 / 19)$ and $\nu_{\mathrm{QE}}=1 / 5(\nu=6 / 17)$ states should be very small, which agrees very well with exact few electron calculations. We believe that taking into account the behavior of involved QP pseudopotentials on all levels of hierarchy should explain all observed odd denominator FQH fillings and allow prediction of their relative stability (without using trial wave functions involving multiple LL's and projections onto the lowest LL needed in the Jain [5] picture).

\section{Conclusion}

Using the pseudopotential formalism, we described the FQH states in terms of the ability of electrons to avoid strongly repulsive pair states. We defined the class of SR pseudopotentials leading to the formation of incompressible FQH states. We argue that the MFCF picture is justified for the SR interactions and fails for others. The pseudopotentials of the Coulomb interaction in excited LL's and of the Laughlin QP's in the $\nu=1 / 3$ state are shown to belong to the SR class only at certain filling factors. 


\section{Acknowledgment}

This work has been supported in part by the Materials Research Program of Basic Energy Sciences, US Department of Energy. A.W. thanks Witold Bardyszewski (Warsaw University) for help with improving the numerical codes.

\section{References}

[1] K. von Klitzing, G. Dorda, M. Pepper, Phys. Rev. Lett. 45, 494 (1980).

[2] D.C. Tsui, H.L. Störmer, A. C. Gossard, Phys. Rev. Lett. 48, 1559 (1982).

[3] R. Laughlin, Phys. Rev. Lett. 50, 1395 (1983).

[4] The Quantum Hall Effect, Eds. R.E. Prange, S.M. Girvin, Springer-Verlag, New York 1987.

[5] J. Jain, Phys. Rev. Lett. 63, 199 (1989).

[6] A. Lopez, E. Fradkin, Phys. Rev. B 44, 5246 (1991).

[7] F.D.M. Haldane, E.H. Rezayi, Phys. Rev. Lett. 60, 956 (1988); F.D.M. Haldane, Ch. 8 in Ref. [4].

[8] A. Wójs, J.J. Quinn, Solid State Commun. 108, 493 (1998); ibid. 110, 45 (1999); Phys. Rev. B, submitted for publication, cond-mat/9903145.

[9] A. de Shalit, I. Talmi, Nuclear Shell Theory, Academic Press, New York 1963; R.D. Cowan, The Theory of Atomic Structure and Spectra, University of California Press, Berkeley 1981.

[10] F.D.M. Haldane, Phys. Rev. Lett. 51, 605 (1983).

[11] P. Sitko, K.-S. Yi, J.J. Quinn, Phys. Rev. B 56, 12417 (1997).

[12] A. Wójs, P. Hawrylak, J.J. Quinn, Physica B 256-258, 490 (1998); Phys. Rev. B, in press; A. Wójs, I. Szlufarska, K.-S. Yi, J.J. Quinn, Phys. Rev. B, in press.

[13] T.T. Wu, C.N. Yang, Nucl. Phys. B 107, 365 (1976).

[14] X.M. Chen, J.J. Quinn, Solid State Commun. 92, 865 (1996).

[15] P. Sitko, S.N. Yi, K.-S. Yi, J.J. Quinn, Phys. Rev. Lett. 76, 3396 (1996).

[16] R. Willet, J.P. Eisenstein, H.L. Störmer, D.C. Tsui, A.C. Gossard, J.H. English, Phys. Rev. Lett. 59, 1776 (1987). 